\title{
Late recurrence of a malignant hypoglycemia-inducing pelvic solitary fibrous tumor secreting high-molecular-weight insulin-like growth factor-II: A case report with protein analysis
}

\author{
HIROKI ISHIHARA $^{1}$, KENJI OMAE ${ }^{1}$, JUNPEI IIZUKA ${ }^{1}$, HIROHITO KOBAYASHI ${ }^{1}$, IZUMI FUKUDA ${ }^{2}$, \\ TSUNENORI KONDO ${ }^{1}$, NAOMI HIZUKA ${ }^{2}$, YOJI NAGASHIMA ${ }^{3}$ and KAZUNARI TANABE ${ }^{1}$ \\ Departments of ${ }^{1}$ Urology, ${ }^{2}$ Hypertension and Endocrinology and ${ }^{3}$ Pathology, \\ Tokyo Women's Medical University, Tokyo 162-8666, Japan
}

Received March 23, 2015; Accepted May 17, 2016

DOI: $10.3892 / \mathrm{ol} .2016 .4665$

\begin{abstract}
The present study reports a case of recurrent malignant pelvic solitary fibrous tumor (SFT) that induced non-islet cell tumor hypoglycemia via high-molecular-weight insulin-like growth factor-II in a 72-year-old male patient. The tumor recurred $\sim 12$ years after the complete resection of the original mass. The recurrent tumor, which had directly invaded the left ureter and perirectal fat tissue, could not be completely excised due to its fragility and adhesiveness. At 13 days post-surgery, the patient presented with rectal perforation, and an urgent rectal resection and colostomy was performed. Neither recurrence of the tumor nor hypoglycemic symptoms were observed 9 months after the surgery. High molecular weight insulin-like growth factor-II was detected in the serum and tumor specimens by western blot analysis and immunohistochemistry. The present case report suggests that certain SFTs can relapse even $\geq 10$ years after a presumed complete resection of the primary tumor, and that performing a safe and complete resection of these tumors can be challenging, due to their adhesiveness or physical presentation; therefore, the indications for surgery should be considered with caution.
\end{abstract}

\section{Introduction}

Solitary fibrous tumors (SFTs) are rare, non-functional, and generally benign mesenchymal tumors, with an estimated

Correspondence to: Dr Kenji Omae, Department of Urology, Tokyo Women's Medical University, 8-1 Kawada-cho, Shinjuku-ku, Tokyo 162-8666, Japan

E-mail: omae@kc.twmu.ac.jp

Abbreviations: SFT, solitary fibrous tumor; NICTH, non-islet cell tumor hypoglycemia; IGF, insulin-like growth factor; CT, computed tomography; GH, growth hormone; HMW, high-molecular-weight

Key words: solitary fibrous tumor, late recurrence, hypoglycemia, insulin-like growth factor, malignant age-standardized incidence of 1.4 per million population $(1,2)$. Despite the fact that SFTs are mainly intrathoracic (1), on rare occasions they can appear in extrapleural sites, such as the retroperitoneal (3) and pelvic regions (4). In total, 10-20\% of SFTs are classified as malignant, and eventually lead to mortality (1). Previous studies have reported the association of malignant SFTs with non-islet cell tumor hypoglycemia (NICTH) due to the secretion of insulin-like growth factor (IGF)-II by the tumor cells $(1,3,5,6)$. Furthermore, certain SFTs have been shown to recur $>10$ years after the resection of the original tumor, regardless of whether the original tumors were benign or malignant (7).

The present study reports a case of malignant pelvic SFT with NICTH due to IGF-II secretion in a 72-year-old male patient. The tumor recurred $\sim 12$ years after the first surgery, despite the presumed complete excision of the original tumor, which is a rare occurrence. The tumor was evaluated using several imaging tests, as well as pathological, immunohistochemical and western blot analyses. A second surgery was performed for the excision of the recurrent tumor, which may have been incomplete due to the tumor's strong adhesiveness and anatomical features. A postoperative computed tomography (CT) scan showed no evidence of either recurrence or metastasis, and, at the time of writing, the patient remained asymptomatic at 9 months post-surgery.

\section{Case report}

A 72-year-old male patient with intermittent loss of consciousness was admitted to another hospital. Laboratory data revealed a reduced blood glucose level of $28 \mathrm{mg} / \mathrm{dl}$ (normal level, $75-109 \mathrm{mg} / \mathrm{dl}$ ), and the patient was deemed to be in a hypoglycemic coma. Subsequent hypoglycemic attacks occurred frequently, necessitating a more extensive examination. Hormonal tests revealed elevated levels of serum IGF-II by western blotting (molecular weight, $20.9 \mathrm{kDa}$ ). Other markers were found to be suppressed, including serum C-peptide $(0.2 \mathrm{ng} / \mathrm{ml}$; normal level, $1.1-3.3 \mathrm{ng} / \mathrm{ml})$, IGF-I $(52.0 \mathrm{ng} / \mathrm{ml}$; normal level, 63.0-206.0 ng/ml), growth hormone (GH) $(<0.05 \mathrm{ng} / \mathrm{ml}$; normal level, $<0.42 \mathrm{ng} / \mathrm{ml})$ and immunoreactive insulin $(<0.1 \mu \mathrm{U} / \mathrm{ml}$; normal level, $<0.4 \mu \mathrm{U} / \mathrm{ml})$. 
A

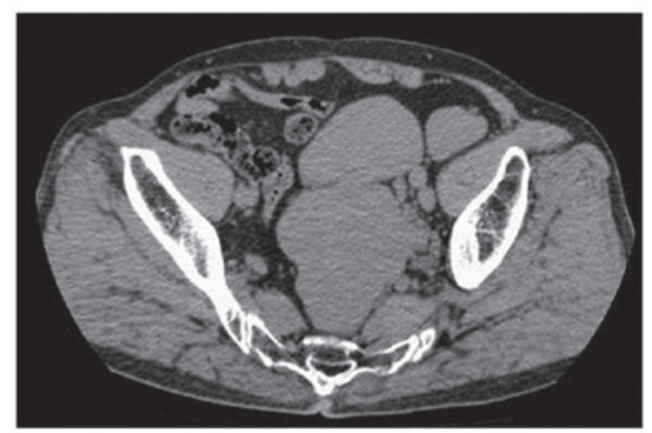

C

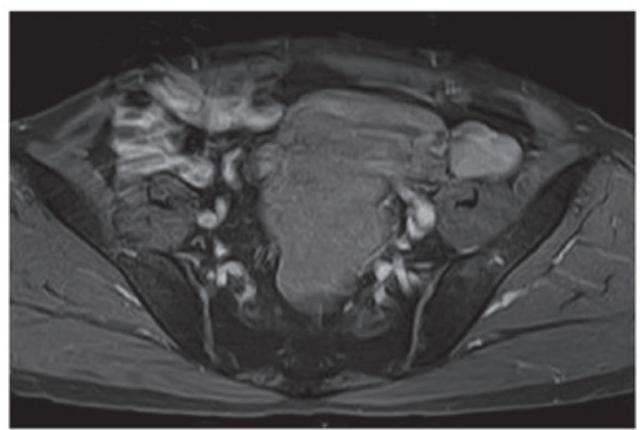

B

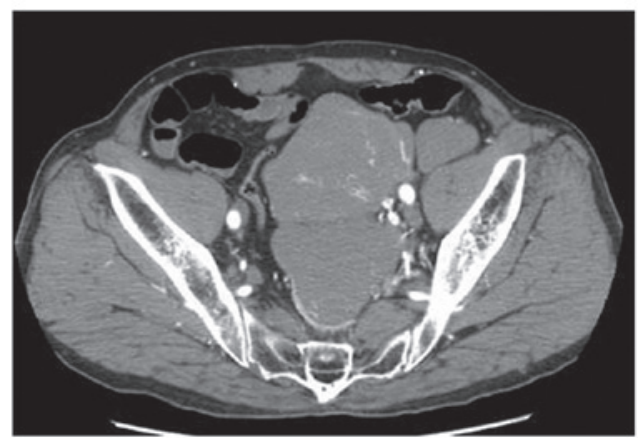

D

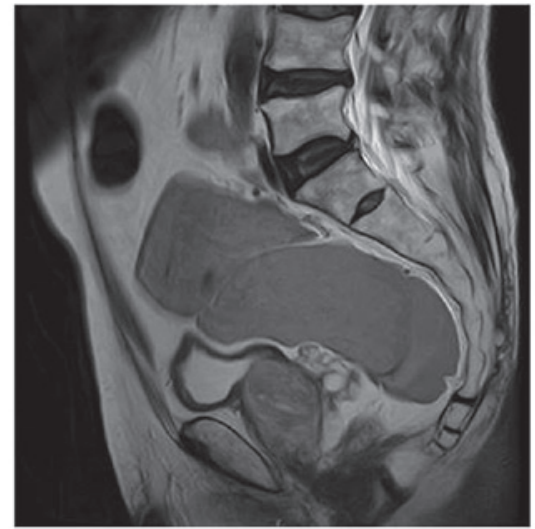

Figure 1. CT and MRI scans. (A) Plain CT. (B) Contrast-enhanced CT; arterial-phase. (C) Plain MRI; T1-weighted sequence. (D) Plain MRI; T2-weighted sequence. The CT scan showed a huge homogenous tumor occupying the pelvis. The tumor exhibited little contrast enhancement, and consisted of 3 main components that were located ventrally to the sacral bone ( 78x101x67 mm), immediately above the bladder ( 85x99x62 mm) and ventrally to the left common iliac artery $(\sim 49 \times 21 \times 32 \mathrm{~mm})$. The MRI scan revealed a mostly homogenous tumor in the pelvis with little contrast enhancement. CT, computed tomography; MRI, magnetic resonance imaging.

Abdominal CT and magnetic resonance imaging (MRI) scans revealed a pelvic tumor measuring $\sim 7 \mathrm{~cm}$ in diameter. On the basis of these findings, an IGF-producing tumor was suspected. Primary surgery was performed on May 2002, and the tumor was completely excised. Microscopically, the tumor was diagnosed as an IGF-II-producing SFT, based on its positive immunoreactivity for cluster of differentiation 34 (CD34) and IGF-II. Furthermore, partial hemorrhagic and necrotic findings, as well as high mitotic activity, suggested a malignant phenotype. The tumor was ultimately diagnosed as a malignant pelvic SFT with NICTH due to IGF-II, and regular follow-up CT scans and laboratory examinations were performed for 5 years, with no evidence of tumor recurrence or hypoglycemia, which were then discontinued at the patient's own prerogative.

Approximately 12 years later, the patient began to re-experience episodes of loss of consciousness. Laboratory examinations revealed low blood glucose level (40 mg/dl), and an enhanced CT scan revealed a poorly-enhanced homogeneous tumor measuring $\sim 90 \mathrm{~mm}$ in diameter, spreading within the pelvis. The tumor appeared to consist of 3 major components that were located ventrally to the sacral bone, immediately above the bladder and ventrally to the left common iliac artery (Fig. 1A and B). Pelvic SFT recurrence was suspected, and the patient was referred to the Department of Urology, Tokyo Women's Medical University (Tokyo, Japan) on June 2014 for further evaluation and treatment. Endocrinological tests revealed that the levels of $\mathrm{GH}$ and IGF-I were suppressed (GH, <0.03 ng/ml; IGF-I, $27 \mathrm{ng} / \mathrm{ml}$ ).
An abdominal MRI scan showed a homogenous tumor with a low contrast-enhancement occupying the pelvis. T2-weighted images revealed a tumor that was iso- to hyper-intense with low diffusion (Fig. 1C and D), while the T1-weighted images demonstrated that the tumor had clear margin. In addition, 18F-fluorodeoxyglucose (FDG)-positron emission tomography/CT was performed (Fig. 2). The maximal intensity projection image showed a moderate FDG uptake in the pelvic tumor (Fig. 2A). The maximum standardized uptake value was 3.37 (Fig. 2B and D), 4.56 (Fig. 2B and D) and 4.16 (Fig. 2C) in each compartment of the tumor located ventral to the sacral bone, right above the bladder, and ventral to the left common iliac artery, respectively.

The tumor was diagnosed as a recurrent pelvic SFT and a second surgery was performed. Intraoperatively, the tumor was found to be fairly fragile and adhesive, and to have directly invaded the left ureter and perirectal fat tissue. The maximum amount of tumor possible was removed. The resected tumor tissue was immediately fixed with $20 \%$ formalin, embedded in paraffin, and subjected to histopathological diagnosis and immunohistochemistry. Paraffin sections (4- $\mu$ m-thick) were stained with hematoxylin and eosin for routine histopathological diagnosis. Macroscopically, the tumor was grayish-white with prominent hemorrhage and necrosis (Fig. 3). Pathologically, the tumor revealed mild-sized spindle cells, densely arranged along a collagen background, with round and oval nuclei and a fine granular eosinophilic cytoplasm (Fig. 4A and B).

For immunohistochemistry, the paraffin sections were stained using an autostainer (Ventana Medical Systems, Inc., 
A

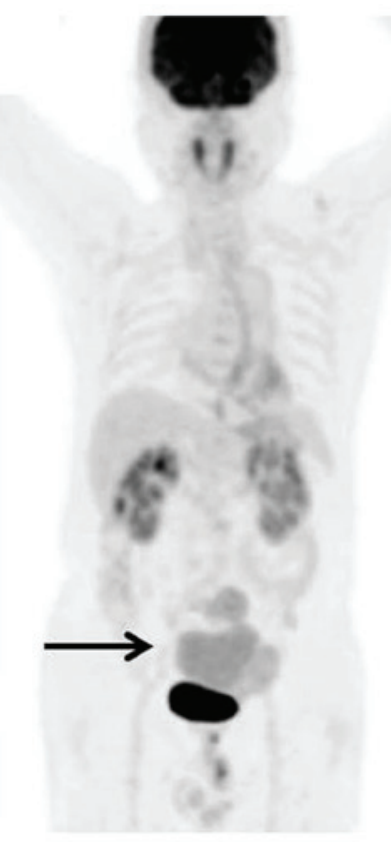

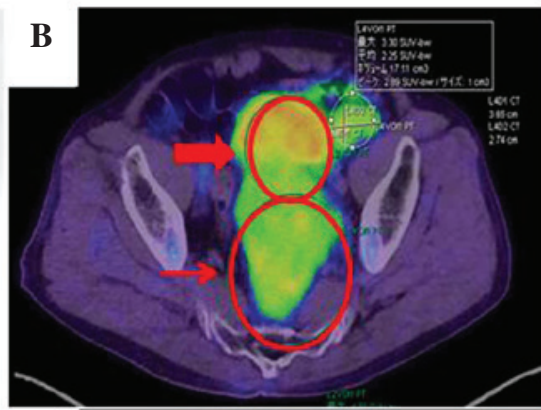

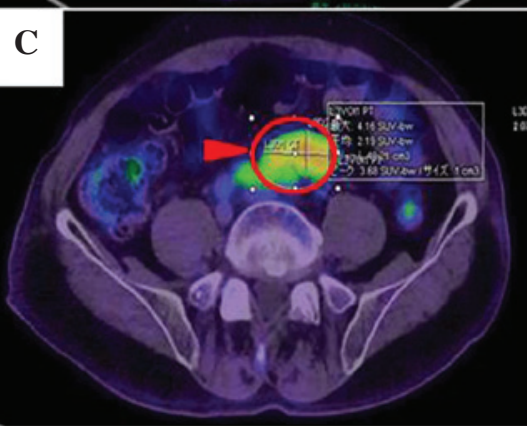

D

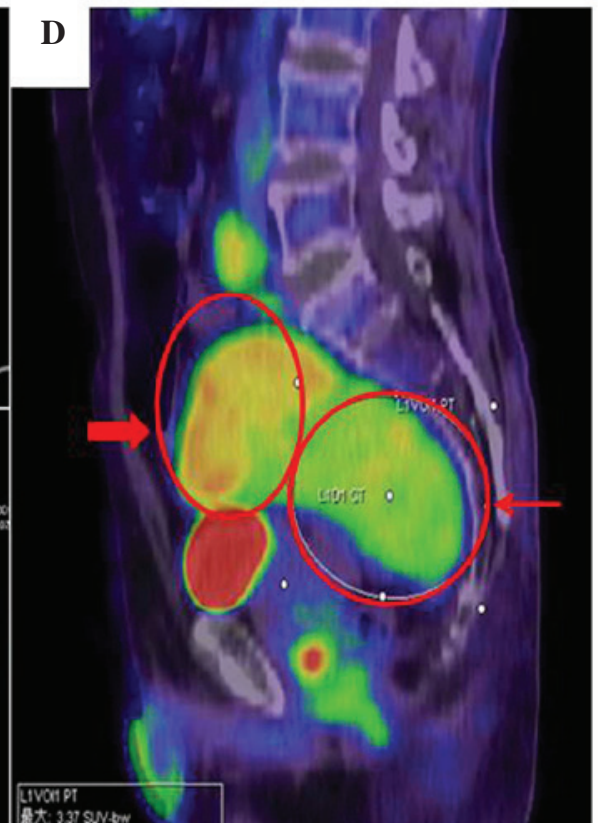

Figure 2. FDG-PET/CT findings. (A) The FDG PET/CT scan showed moderate FDG accumulation in the pelvic tumor on maximal intensity projection. (B) Axial PET/CT image. (C) Axial PET/CT image. (D) Sagittal PET/CT image. The maximum standard uptake values were (B and D; thin arrow) 3.37, (B and D; thick arrow) 4.56 and (C; arrowhead) 4.16 in the pelvic tumors located ventral to the sacral bone, right above the bladder,and ventral to the left common iliac artery, respectively. FDG, 18F-fluorodeoxyglucose; PET, positron emission tomography; CT, computed tomography.

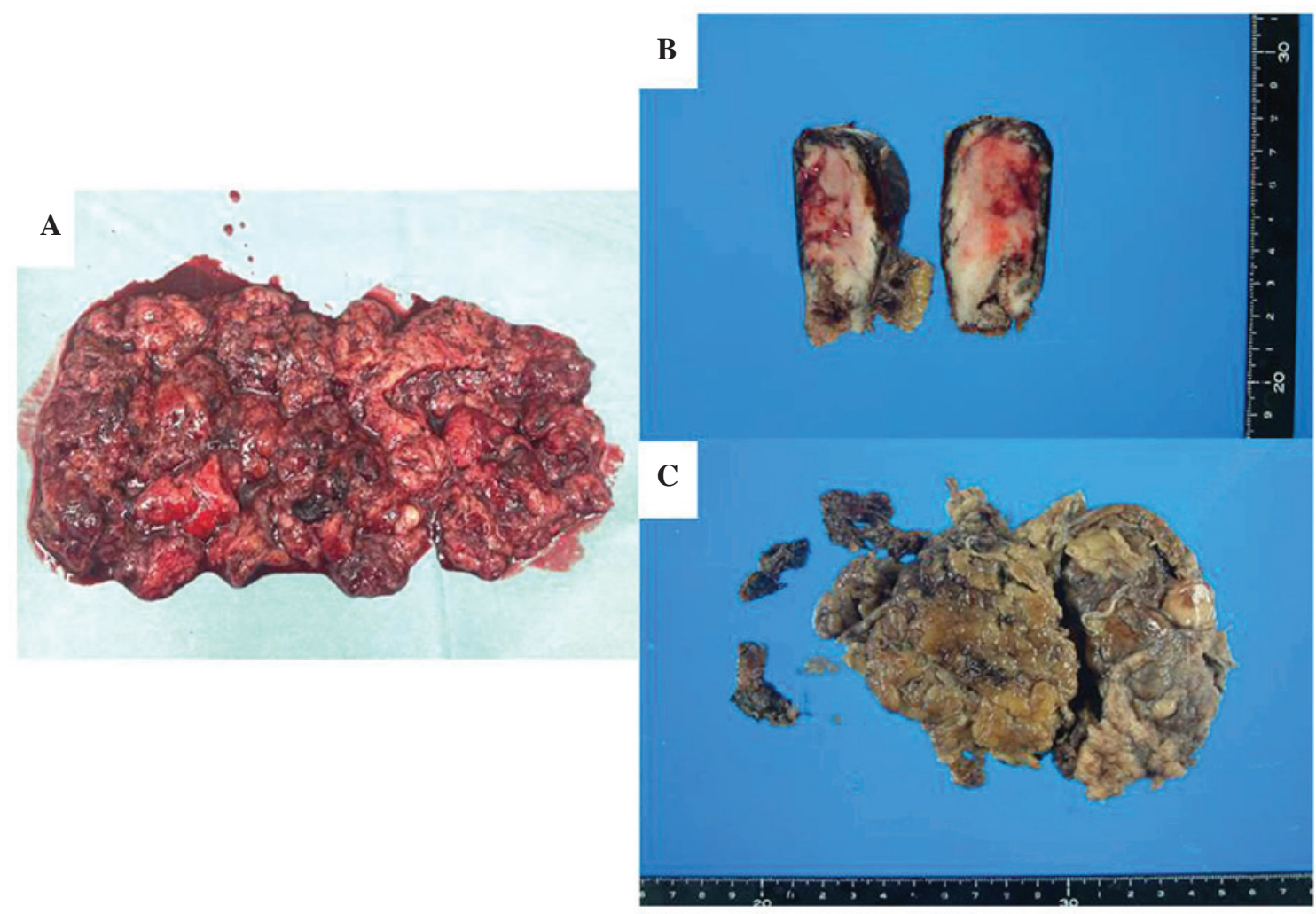

Figure 3. Macroscopic findings. (A) Gross specimen was generally fragile and prone to collapse. Sectioning revealed a grayish-white mass with prominent (B) hemorrhaging and (C) necrosis. Surgical margins were largely ambiguous.

Tucson, AZ, USA). Briefly, sections were deparaffinized in xylene, rehydrated in graded ethanol and immersed in $0.3 \%$ hydrogen peroxide to quench the intrinsic peroxidase. Following incubation with normal sera of the animals in which the secondary antibodies were raised, antigen retrieval was performed by autoclaving the samples in Tris-ethylenediaminetetraacetic acid buffer $(\mathrm{pH} 9.0)$ in a pressure cooker. Subsequently, the samples were incubated with the following primary antibodies diluted 200-fold: Anti-B-cell lymphoma 2 (bcl-2) (clone 124; Dako, Glostrup, Denmark), 


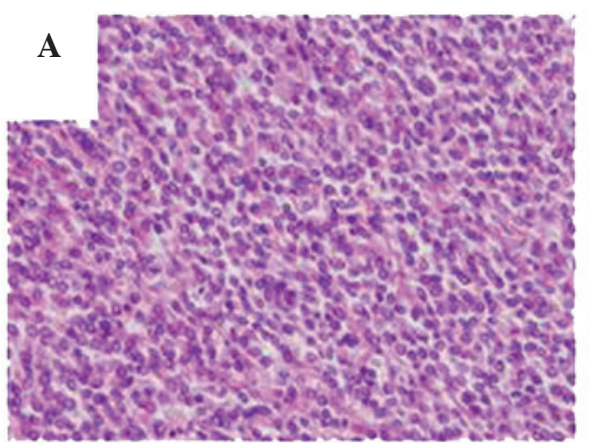

$\mathrm{HE} \times 40$

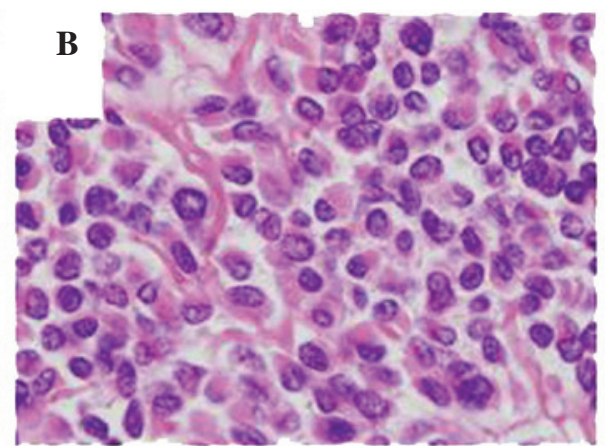

$\mathrm{HE} \times 100$

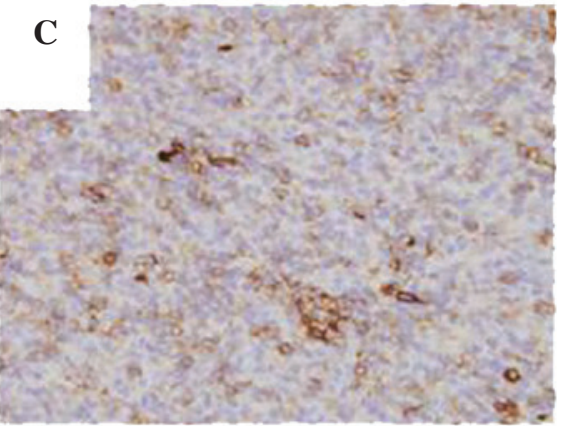

CD 34

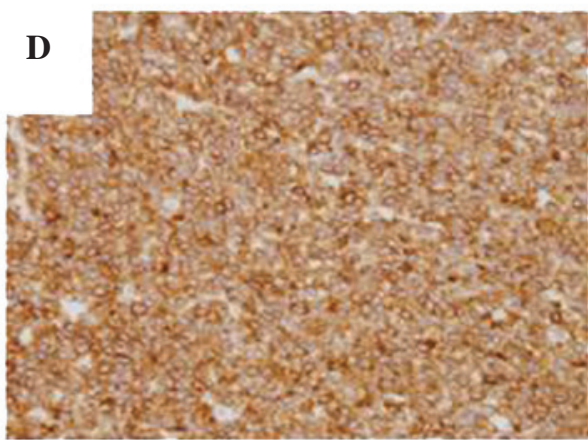

bcl-2

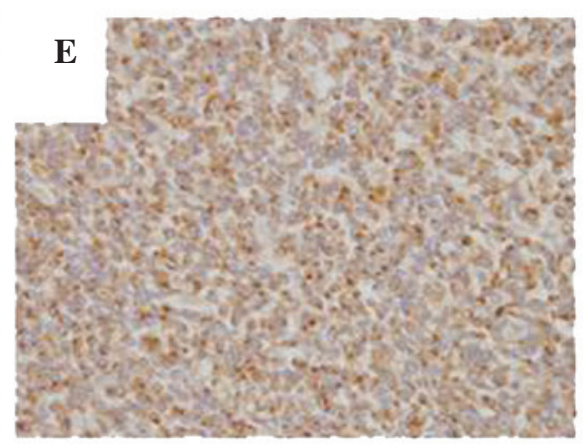

IGF- II

Figure 4. Immunohistochemical findings. Microscopic examinations revealed that the tumor was composed of mild-sized spindle cells densely arranged on a collagen background, with round and oval nuclei and a fine granular eosinophilic cytoplasm; hematoxylin and eosin staining; magnification, (A) x40 and (B) x 100). Immunohistochemical staining for (C) CD34 and (D) bcl-2 showed diffuse positive staining. (E) Immunohistochemical staining for IGF-II showed positive staining across the majority of the tumor cells. Immunoreactivity was also positive for vimentin and negative for c-kit and S-100. The Ki-67 proliferative index was $<5 \%$ (data not shown). CD34, cluster of differentiation 34; bcl-2, B-cell lymphoma 2; IGF-II, insulin-like growth factor-II.

anti-CD34 (clone QBEnd 10; Dako) and anti-IGF-II (clone S1F2; Upstate Biotechnology, Inc., Lake Placid, NY, USA). Subsequently, the sections were rinsed with phosphatebuffered saline, and treated with biotinylated secondary antibodies and streptavidin-conjugated horseradish peroxidase. The labeled antigens were visualized using Ventana Universal DAB kit (catalog no., 518-100431; Ventana Medical Systems, Inc.), and the sections were counterstained with hematoxylin. The tumor cells were immunohistologically positive for IGF-II, CD34, bcl-2 and vimentin, but negative for c-kit and S-100. The Ki-67 proliferative index was $<5 \%$ (Fig. 4C-E). Abnormal mitosis was mild and the Ki-67 proliferative index was low; however, according to two pathologists of the Department of Pathology, Tokyo Women's Medical University, the tumor appeared to be malignant due to its hypercellularity, infiltrative growth, gross-necrosis and cellular atypia.

Western blot analysis was performed by the Department of Hypertension and Endocrinology of Tokyo Women's Medical University to investigate the preoperative heterogeneity of serum IGF-II forms and the retention of IGF-II postoperatively (Fig. 5). As shown in Fig. 5 (lane 2), the patient's serum contained the high molecular weight (HMW)-form of IGF-II. This result was similar to the results of other tumors secreting IGF-II, obtained from previously studied patients (Fig. 5; lanes 5 and 6). HMW-IGF-II disappeared 4 days following the procedure, although several other bands appeared (Fig. 5; lane 3). At 13 days post-surgery, the patient presented with

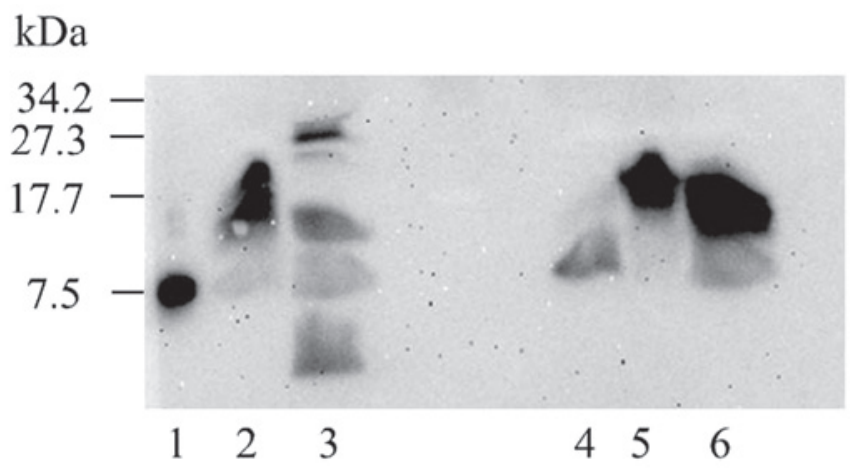

Figure 5. Western blot analysis of serum IGF-II. Western blot analysis revealed that the preoperative IGF-II in the patient's serum was of the HMW-form (lane 2), which is consistent with previously studied patients with non-islet cell tumor hypoglycemia due to an IGF-II producing tumor (lanes 5 and 6). Western blot analysis also showed that HMW-IGF-II disappeared 4 days after the procedure (lane 3 ), but several unidentified bands appeared. In a patient from the healthy control (lane 4), slight amounts of IGF-II fat $(7.5 \mathrm{kDa})$ were detected. IGF-II, insulin-like growth factor-II; HMW, high-molecular-weight.

rectal perforation, and a rectal resection and colostomy were immediately performed. Since the tumor was found to be adhesive and to have directly invaded the left ureter and perirectal fat tissue, long-time and careful observations were required to ensure that no tumor traces remained after surgery. Thus, the patient was followed up by blood examination every 
1 or 2 months, and by imaging examination such as CT every 3 months. No tumor recurrence or hypoglycemic symptoms were observed on the follow-up by blood examination every 1 or 2 months and imaging examination, such as CT scan, every 3 months for 9 months after the surgery, and no further treatments were performed.

\section{Discussion}

Certain patients with SFTs experience frequent hypoglycemic episodes, which are associated with IGF-II production. It has been reported that, while $\sim 4 \%$ of pleural SFTs are associated with hypoglycemia, the incidence rate of hypoglycemia in retroperitoneal cases is $\sim 11.5 \%(1,3,5)$. While high levels of IGF-II are generally detected in the serum or tumor cells, Daughaday et al (6) reported an increase in the HMW form of IGF-II in patients with NICTH. The screening and detection of suppressed IGF-I levels and increased serum IGF-II/IGF-I ratios may serve as useful diagnostic markers (8). A deficiency in functional GH could serve as a secondary marker for NICTH. Low IGF-I levels in patients with NICTH are attributed to chronic attenuation of $\mathrm{GH}$ secretion, due to the negative feedback of IGF-II (9). Several studies have shown that NICTH due to HMW-IGF-II can be diagnosed using western blot analysis (10-12). Hata et al (10) examined HMW-IGF-II by using preoperative and postoperative blots and demonstrated that HMW-IGF-II disappeared postoperatively, which is considered to be a useful therapeutic index. In the present case, perioperative western blot analysis was also performed, which revealed the disappearance of HMW-IGF-II, but also the appearance of several unidentified protein bands. We speculated that this result may have been due to either hemolysis in the blood samples, artifacts caused by obtaining the postoperative sample too soon, or incomplete removal of the tumor.

Surgical removal is considered the gold standard for the treatment of SFTs $(13,14)$. Resectability is the most important factor influencing the outcome, and complete excision should allow for a favorable prognosis without recurrence or metastasis, even if the tumor is malignant (15). However, Baldi et al (7) reported a number of SFT cases that relapsed $>10$ years after initial diagnosis, despite a history of surgical excision of the primary tumor. Additionally, it is possible that certain inoperable cases are encountered, which are due to a poor general condition of the patient, metastasis or severe infiltration to other tissues and organs. Several studies have indicated that the length of time between the first and second recurrence is shorter compared with the time between the initial diagnosis and the first recurrence in frequently-recurring SFTs, regardless of whether surgery was performed $(7,10)$. Baldi et al (7) suggested that the inherent limitations of surgery may result in unavoidable recurrence or metastasis, since the anatomy of the retroperitoneum and pelvis make complete resection challenging. Thus, high rates of local failure could occur even for benign SFTs, due to incomplete removal. Frequent surgical resection for recurrent tumors causes aggressive adhesion, making complete surgical resection a considerable challenge. Furthermore, incomplete resection may actually cause tumor dissemination. These factors may explain why the time between the first and second recurrence is shorter. Thus, intraoperative findings and tumor location should be considered an associated risk for recurrence or metastasis in conjunction with pathological findings. In the present case, intraoperative findings and postoperative western blot analysis results indicated that a second recurrence will most likely occur more quickly than the first recurrence.

Although there are currently no consensus guidelines for curative treatments other than surgery, several previous studies have reported other effective treatments. Hosaka et al (16) reported that a malignant pelvic IGF-II-secreting SFT was successfully treated via intra-arterial chemotherapy with cisplatin and carboplatin and concurrent radiotherapy for 5 years. Although the patient had NICTH prior to treatment (as in the present case), hypoglycemic attacks were resolved in tandem with tumor shrinkage (16). Other studies reported that glucocorticoid therapy may be effective against hypoglycemia caused by IGF-II-producing tumors or tumor growth itself $(11,17)$. Furthermore, several studies have suggested that imatinib mesylate is an effective therapy for unresectable SFTs $(12,18,19)$. Yamada et al $(20)$ reported that the Akt-mammalian target of rapamycin pathway is activated in $~ 50 \%$ of SFTs and is associated with the upregulation of receptor tyrosine kinases. Furthermore, sunitinib malate may be efficacious due to its anti-platelet-derived growth factor receptor- $\beta$ activity (21). Recent genetic research revealed that the recurrent fusion of two genes, NGFI-A binding protein 2 and signal transducer and activator of transcription 6 , both located at chromosomal region $12 \mathrm{q} 31$, was identified as a SFT-specific chimeric fusion gene by next-generation sequencing and reverse transcription polymerase chain reaction (22-24). Finally, the development of new-generation drugs that target tumor-promoting genes or proteins is also essential. In the present case, intraoperative findings and western blot analysis results suggested that the resection of the tumor was incomplete, since the tumor was found to be adhesive and to have directly invaded the left ureter and perirectal fat tissue. Thus, adjuvant therapies such as the ones discussed, should be investigated in an effort to prevent recurrence, as current options do not appear to be effective.

In conclusion, the present study reports a rare case of malignant pelvic NICTH-inducing SFT with IGF-II secretion, which recurred $\sim 12$ years after the presumed complete resection of the primary tumor. The present case report suggested that the safe and complete resection of tumors may sometimes be challenging to perform, due to their adhesiveness or physical presentation; therefore, the indications for surgery should be considered with caution.

\section{Acknowledgements}

The authors would like to thank Editage (www.editage.jp) for the English language editing.

\section{References}

1. Briselli M, Mark EJ and Dickersin GR: Solitary fibrous tumors of the pleura: Eight new cases and review of 360 cases in the literature. Cancer 47: 2678-2689, 1981.

2. Thorgeirsson T, Isaksson HJ, Hardardottir H, Alfredsson $\mathrm{H}$ and Gudbjartsson T: Solitary fibrous tumors of the pleura: An estimation of population incidence. Chest 137: 1005-1006, 2010. 
3. Takizawa I, Saito T, Kitamura Y, Arai K, Kawaguchi M, Takahashi K and Hara N: Primary solitary fibrous tumor (SFT) in the retroperitoneum. Urol Oncol 26: 254-259, 2008.

4. Tsushimi T, Yagi T, Tomozawa N and Ohnishi H: Retroperitoneal solitary fibrous tumor of the pelvis with pollakiuria: A case report. BMC Res Notes 5: 593, 2012.

5. Mentzel T, Bainbridge TC and Katenkamp D: Solitary fibrous tumour: Clinicopathological, immunohistochemical, and ultrastructural analysis of 12 cases arising in soft tissues, nasal cavity and nasopharynx, urinary bladder and prostate. Virchows Arch 430: 445-453, 1997.

6. Daughaday WH, Emanuele MA, Brooks MH, Barbato AL, Kapadia M and Rotwein P: Synthesis and secretion of insulin-like growth factor II by a leiomyosarcoma with associated hypoglycemia. N Engl J Med 319: 1434-1440, 1988.

7. Baldi GG, Stacchiotti S, Mauro V, Dei Tos AP, Gronchi A, Pastorino U, Duranti L, Provenzano S, Marrari A, Libertini M, et al: Solitary fibrous tumor of all sites: Outcome of late recurrences in 14 patients. Clin Sarcoma Res 3: 4, 2013.

8. Fukuda I, Hizuka N, Ishikawa Y, Yasumoto K, Murakami Y, Sata A, Morita J, Kurimoto M, Okubo Y and Takano K: Clinical features of insulin-like growth factor-II producing non-islet-cel tumor hypoglycemia. Growth Horm IGF Res 16: 211-216, 2006.

9. Ron D, Powers AC, Pandian MR, Godine JE and Axelrod L: Increased insulin-like growth factor II production and consequent suppression of growth hormone secretion: A dual mechanism for tumor-induced hypoglycemia. J Clin Endocrinol Metab 68 701-706, 1989.

10. Hata T, Tsuruta Y, Takamori S and Shishikura Y: Non-islet cell tumor hypoglycemia at the second recurrence of malignant solitary fibrous tumor in the retroperitoneum and pelvis: A case report. Case Rep Oncol 5: 420-427, 2012.

11. Tsuro K, Kojima H, Okamoto S, Yoshiji H, Fujimoto M, Uemura M, Yoshikawa M, Nakamura T, Kou S, Nakajima Y and Fukui H: Glucocorticoid therapy ameliorated hypoglycemia in insulin-like growth factor-II-producing solitary fibrous tumor. Intern Med 45: 525-529, 2006.

12. Tominaga N, Kawarasaki C, Kanemoto K, Yokochi A, Sugino K, Hatanaka K, Uekusa T, Fukuda I, Aiba M, Hizuka N and Uda S: Recurrent solitary fibrous tumor of the pleura with malignant transformation and non-islet cell tumor-induced hypoglycemia due to paraneoplastic overexpression and secretion of high-molecular-weight insulin-like growth factor II. Intern Med 51: 3267-3272, 2012

13. Morimitsu Y, Nakajima M, Hisaoka $M$ and Hashimoto $H$ : Extrapleural solitary fibrous tumor: Clinicopathologic study of 17 cases and molecular analysis of the p53 pathway. APMIS 108: $617-625,2000$
14. Brunnemann RB, Ro JY, Ordonez NG, Mooney J, El-Naggar AK and Ayala AG: Extrapleural solitary fibrous tumor: A clinicopathologic study of 24 cases. Mod Pathol 12: 1034-1042, 1999.

15. England DM, Hochholzer L and McCarthy MJ: Localized benign and malignant fibrous tumors of the pleura. A clinicopathologic review of 223 cases. Am J Surg Pathol 13: 640-658, 1989.

16. Hosaka S, Katagiri H, Wasa J, Murata $\mathrm{H}$ and Takahashi $\mathrm{M}$ Solitary fibrous tumor in the pelvis: Induced hypoglycemia associated with insulin-like growth factor II. J Orthop Sci 20: 439-443, 2015

17. Pavelić K, Vrbanec D, Marusić S, Levanat S and Cabrijan T: Autocrine tumour growth regulation by somatomedin $\mathrm{C}$ An in-vitro model. J Endocrinol 109: 233-238, 1986.

18. Prunotto M, Bosco M, Daniele L, Macri' L, Bonello L, Schirosi L, Rossi G, Filosso P, Mussa B and Sapino A: Imatinib inhibits in vitro proliferation of cells derived from a pleural solitary fibrous tumor expressing platelet-derived growth factor receptor-beta. Lung Cancer 64: 244-246, 2009.

19. De Pas T, Toffalorio F, Colombo P, Trifirò G, Pelosi G, Vigna PD, Manzotti M, Agostini M and de Braud F: Brief report: Activity of imatinib in a patient with platelet-derived-growth-factor receptor positive malignant solitary fibrous tumor of the pleura. J Thorac Oncol 3: 938-941, 2008.

20. Yamada Y, Kohashi K, Fushimi F, Takahashi Y, Setsu N, Endo M, Yamamoto H, Tokunaga S, Iwamoto Y and Oda Y: Activation of the Akt-mTOR pathway and receptor tyrosine kinase in patients with solitary fibrous tumors. Cancer 120: 864-876, 2014.

21. Stacchiotti S, Negri T, Palassini E, Conca E, Gronchi A, Morosi C, Messina A, Pastorino U, Pierotti MA, Casali PG and Pilotti S: Sunitinib malate and figitumumab in solitary fibrous tumor: Patterns and molecular bases of tumor response. Mol Cancer Ther 9: 1286-1297, 2010.

22. Barthelmeß S, Geddert H, Boltze C, Moskalev EA, Bieg M, Sirbu H, Brors B, Wiemann S, Hartmann A, Agaimy A and Haller F: Solitary fibrous tumors/hemangiopericytomas with different variants of the NAB2-STAT6 gene fusion are characterized by specific histomorphology and distinct clinicopathological features. Am J Pathol 184: 1209-1218, 2014.

23. Robinson DR, Wu YM, Kalyana-Sundaram S, Cao X, Lonigro RJ, Sung YS, Chen CL, Zhang L, Wang R, Su F, et al: Identification of recurrent NAB2-STAT6 gene fusions in solitary fibrous tumor by integrative sequencing. Nat Genet 45: 180-185, 2013.

24. Chmielecki J, Crago AM, Rosenberg M, O'Connor R, Walker SR, Ambrogio L, Auclair D, McKenna A, Heinrich MC, Frank DA and Meyerson M: Whole-exome sequencing identifies a recurrent NAB2-STAT6 fusion in solitary fibrous tumors. Nat Genet 45: 131-132, 2013. 